\title{
Future perfect
}

\section{Abundant frontiers at the interface of chemistry and biology promise another decade of technological innovation and scientific discovery by chemical biologists.}

Since our June issue, in celebration of the tenth anniversary of Nature Chemical Biology, we have published a series of articles highlighting the scientific achievements of chemical biology and featuring the viewpoints of chemical biologists on their evolving field (Nat. Chem. Biol. 11, 363, 2015). In this last issue of our anniversary volume, we present a collection of articles focused on "Frontiers in chemical biology," which identify some of the emerging scientific areas that will engage chemical biologists in the coming years.

In the final installment of our "Voices of chemical biology" series published in this issue (Q\&A, p. 900), we feature thoughts from the community about scientific frontiers in chemical biology and how new technologies will facilitate research in these areas. These perspectives not only illustrate the potential impact of chemical biology in all disciplines at the chemistry-biology interface but also underscore some defining features of the field, including a motivation to develop more sophisticated and versatile tools, a desire to discover new biology and understand molecular mechanisms in increasingly complex biological systems, and an aspiration to design new chemical or biological systems with tailored properties (Nat. Chem. Biol. 11, 363, 2015).

Independent of the scientific question being addressed, chemical biology is uniquely positioned to offer the smallmolecule and biomolecular tools needed to visualize, measure and control biological pathways in situ with unprecedented precision. For instance, as noted by Johnsson and colleagues (Perspective, p. 917), the development of chemical approaches to new synthetic probes for live-cell imaging and for endowing proteins with new functionality will advance current imaging technologies and enable a more complete understanding of cellular pathways.

Chemical probes, small molecules that potently modulate the activities of specific targets within cells, represent canonical chemical biology tools that have illuminated the molecular details of numerous biological pathways (see, for example, p. 973). Chemical probes will assume an increasingly important role in frontier research at the interfaces of chemistry, biology and medicine (Nat. Chem. Biol. 11, 533, 2015), particularly in expanding the druggable genome to targets that were previously considered intractable (Nat. Rev. Drug Disc. 1, 727-730, 2002). For instance, in a Review in this issue (p. 924), Brown and Stokes propose that specific chemical probes developed to target ribosome assembly factors and key protein-protein interactions will become essential tools for studying ribosome biogenesis and identify new ways to target translation. Similarly, Mapp and colleagues (Commentary, p. 891) argue that selective targeting of transcription is now within reach, supported by our more sophisticated understanding of proteinprotein interactions and our improved ability to craft molecules to target these interfaces. Taken together, these articles suggest a promising future for efforts to precisely modulate the compositions and activities of cellular protein complexes.

Identifying the cellular targets of chemical probes remains an essential step in translating basic science discoveries in chemical biology to the clinic, and chemical biologists remain at the forefront of efforts to develop new target identification methods. Nijman (Review, p. 942) describes recent applications of functional genomic approaches, including CRISPR-Cas9 targeting, haploid cell line screening and resistance mutation mapping by nextgeneration sequencing, that represent new frontier technologies for small-molecule target discovery.

As a basic science discipline, chemical biology will continue to be a driver for biological discovery and provide means to characterize the molecular bases of these new pathways. Saghatelian and Couso (Perspective, p. 909) illustrate this point with the example of short open reading frame-encoded polypeptides (SEPs), a class of ribosomally expressed bioactive peptides that was recently uncovered within genomes. After reviewing the discovery and biological functions of known SEPs, the authors suggest that chemical biologists will have a unique role in understanding peptidomic space and elucidating the likely diverse functions of SEP action in cells.

The "structure-function relationship"the link between a molecule's structure and its function (activity or reactivity) - is a foundational principle in chemical biology that informs our understanding of biochemical mechanisms and guides our molecular design strategies. For this reason, structural approaches, including NMR and X-ray crystallography, are pervasive tools in the field. Recent advances in structural biology methods, including cryo-electron microscopy (cryo-EM, Nature 525, 172-174, 2015) and serial femtosecond crystallography, which employs X-ray free electron lasers and nanocrystalline samples (see Commentary by Fromme, p. 895), will further enable chemical biologists to arrive at functional mechanistic insights on the basis of structural knowledge.

Despite their potential, current structural approaches have only begun to address the more complex structure-function questions of chemical biologists, such as "What is the structure of this molecule within a living cell and how does its structure engender function?". Spitale and colleagues (Review, p. 933) explore these questions for RNA, by reviewing the evolution of chemical approaches for probing RNA structure in the test tube, and more recently within cells, and highlighting the potential of these reagents to inform the link between dynamic RNA structures and their numerous cellular functions.

Given the diversity of research at the chemistry-biology interface, our anniversary anthology (http://www.nature. com/nchembio/focus/10th_anniversary/) undoubtedly presents only an incomplete snapshot of the exciting frontiers of chemical biology. We hope to look back ten years from now to see that the areas highlighted here have evolved and expanded, but also to be surprised by the emergence of unforeseen frontiers that were driven forward by the creativity and industriousness of chemical biologists.

As our anniversary year draws to a close, we thank the authors who wrote articles for our collection, the referees who provided helpful feedback and all of the contributors to our "Voices of chemical biology" project. Most of all, we thank the chemical biology community for its support of Nature Chemical Biology over the past decade. We look forward to our ongoing responsibility of reflecting the diversity and excitement of frontier chemical biology research as the field defines itself in the years ahead. 